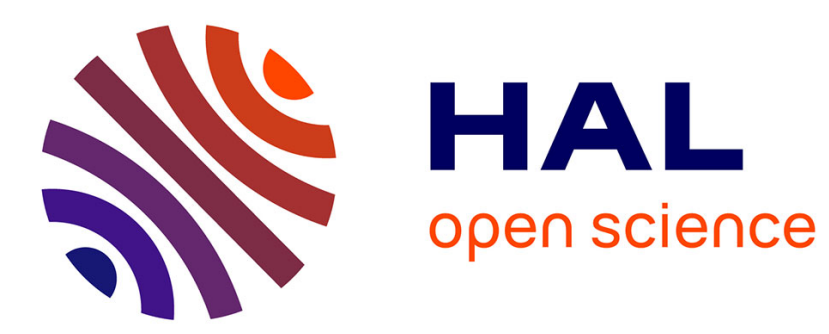

\title{
Closed-loop Central Pattern Generator Control of Human Gaits in OpenSim Simulator
}

\author{
Andrii Dmytrovych Shachykov, Oleksandr Shuliak, Patrick Henaff
}

\section{To cite this version:}

Andrii Dmytrovych Shachykov, Oleksandr Shuliak, Patrick Henaff. Closed-loop Central Pattern Generator Control of Human Gaits in OpenSim Simulator. IJCNN 2019 - International Joint Conference on Neural Networks, Jul 2019, Budapest, Hungary. hal-02309658

\section{HAL Id: hal-02309658 \\ https://hal.science/hal-02309658}

Submitted on 9 Oct 2019

HAL is a multi-disciplinary open access archive for the deposit and dissemination of scientific research documents, whether they are published or not. The documents may come from teaching and research institutions in France or abroad, or from public or private research centers.
L'archive ouverte pluridisciplinaire $\mathbf{H A L}$, est destinée au dépôt et à la diffusion de documents scientifiques de niveau recherche, publiés ou non, émanant des établissements d'enseignement et de recherche français ou étrangers, des laboratoires publics ou privés. 


\title{
Closed-loop Central Pattern Generator Control of Human Gaits in OpenSim Simulator
}

\author{
Andrii Shachykov ${ }^{1}$, Oleksandr Shuliak ${ }^{2}$, and Patrick Hénaff ${ }^{3}$
}

\begin{abstract}
In this paper, a new neuro-musculoskeletal gait simulation platform is presented. This platform is developed to reproduce healthy or altered walking gaits. It is based on an original model of central pattern generator able to generate variable rhythmic signals for controlling biological human leg joints. Output signals of motoneurons are applied to excitation inputs of modelled muscles of the human lower limbs model. Eight central pattern generators control a musculoskeletal model made up of three joints per leg actuated by 44 Hill-type muscle models. Forward dynamics simulation in OpenSim show that it is possible to generate different stable walking gaits by changing parameters of controller. Further work is aimed on development of stable human standing by implementing reflexes.
\end{abstract}

Index Terms-central pattern generator, musculoskeletal model, OpenSim, human walking

\section{INTRODUCTION}

Simulation of human locomotor system helps to deepen our understanding of the human body functioning. More common and complex means of simulation profit the analysis of walk gaits and thus the non-invasive diagnosis.

Computer simulation of physical interaction between environment and body parts with their control requires a musculoskeletal model. Such models of varied rigor provide researchers with biological, bio-mechanical and kinematic data that would be complicated to gather otherwise. This data include time-varied muscle excitation, joint moments, centers of mass and pressure, etc. Simulated musculoskeletal data help to evaluate the motion coordination of a patient and predict its state after a surgical intervention.

Existing musculoskeletal models can simulate realistic locomotion from records (motion capture system) of kinematic and dynamic human data but only in open-loop [1], i.e. without any feedback coming from the environment or the musculoskeletal system itself. Closed-loop simulation requires to consider physiological and environmental feedback and a controller that varies its output depending on this feedback.

The main part of the human motor system is the nervous circuitry that implies neuronal structures located in the brain and in the spinal cord to control muscle activity in several closed loops. These structures are based on neuron

\footnotetext{
${ }^{1}$ Andrii Shachykov is with Université de Lorraine, CNRS, Inria, LORIA, F-54000 Nancy, France and National Technical University of Ukraine "Igor Sikorsky Kyiv Polytechnic Institute", Kyiv, Ukraine andrii.shachykov@loria.fr

${ }^{2}$ Oleksandr Shuliak is with National Technical University of Ukraine "Igor Sikorsky Kyiv Polytechnic Institute", Kyiv, Ukraine shulyak.alex.47@gmail.com

${ }^{3}$ Patrick Hénaff is with Université de Lorraine, CNRS, Inria, LORIA, F54000 Nancy, France patrick.henaffeloria.fr
}

networks located in the brain (basal ganglia, mesencephalic locomotor region (MLR), etc.), oscillating neuron networks of central pattern generators (CPG) located in the spinal cord, and sensory neuron networks that give feedback from body (proprioceptive) and environment (exteroceptive).

To our knowledge, there are few works that comply with both requirements above aiming to build a neuromusculoskeletal simulator working in the closed-loop. The work of Hase et al. [2] was primarily done for purposes of computer animation, though they mentioned possible medical applications. They have used an original musculoskeletal model with Matsuoka oscillators [3] to generate a signal for each degree of freedom, a set of which is transformed for muscles with optimization algorithm. Their work was extended in [4] by adding in-parallel attracting controller to increase robustness of walking motion.

The work of Zhang et al. [5] uses reflex loops with proprioceptive feedback to control a two-muscle system. The aim of the work was to study the peripheral tremor. Jansen et al. [6] study the effect of altered feedback on gait deficits. They use muscle excitations with altered reflex system to control lower-limb musculoskeletal model in forward dynamic simulation. Murai et al. [7] simulate human somatic reflex using proprioceptive feedback to control musculoskeletal model of leg.

The work presented in this paper aims on creating a neuromusculoskeletal simulation platform in OpenSim [1], [8] and able to exhibit various normal or abnormal gaits and based on bio-inspired central pattern generators copying the structures of spinal cord. It extends authors' previous paper [9] which used much simpler musculoskeletal model than OpenSim.

Originality of this paper is characterized in three main ways. First distinct feature is generation of neuronal excitation directly for each group of muscles. Secondly, we implement biologically-plausible afferent feedbacks connected to the CPG, i.e. muscle stretch velocity and non-binary ground pressure force sensing, making a closed-loop simulation of the neuro-musculoskeletal system. And lastly, there is no known neuronal control in OpenSim in a closed loop.

This simulation platform is sought to be able to simulate various gaits, specifically, abnormal gaits of different causes such as neurodegenerative diseases like Parkinson's, muscle degeneration, artificial limbs, surgical interventions, etc. In our approach these causes are simulated by either alternating signal from higher control center in the brain or by changing properties of neuronal model of spinal human gait.

The global gait simulation platform consists of three levels: 


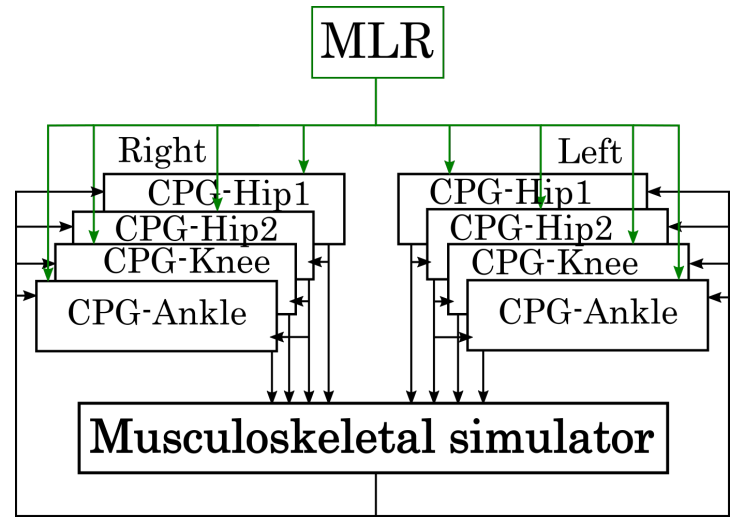

Fig. 1: Scheme of neuronal model of CPG controller. Each CPG controls two antagonistic groups of muscles. In hip joint there are two distinct types of muscles: those who affect only hip joint and those who affect hip and knee joint. They are referred as Hip1 and Hip2 respectively and they influence lower-level CPG.

1) a high-level controller that represents the signals coming from the brain, specifically, MLR;

2) a model of spinal network based on bio-inspired CPG;

3) a musculoskeletal model of human lower limbs executing locomotor movements and producing exteroceptive and proprioceptive feedback.

In this work, the first level acts like a decision-making module that controls start/stop of gait and switches its mode between walk/run. This function is presented as a simple threshold function that inhibits and releases motor function at defined time. Second and third levels are described in Section II which tells about state of the art and methods used in fields of modelling CPG and musculoskeletal system, along with describing our simulation platform. Section III presents obtained simulation results. Section IV concludes this work, with remarks and future work directions.

The results show the ability of our model to simulate a normal healthy gait and a walk interrupted by a sudden stop. Both gaits are controlled by bio-inspired closed-loop CPG based circuitry.

\section{Materials \& Methods}

\section{A. Bio-inspired Central Pattern Generators}

Central pattern generator is a set of inter- and motoneurons located in the spinal cord [10]. They are thought to largely contribute to rhythmic activity such as locomotion, chewing, breathing, etc. [11]. CPG obtain their high-level input through extrapyramidal tracts in brainstem [12]. We will refer to this high-level controller as MLR. This brain region is thought to be the last element in the chain of decision-making regarding autonomous movement.

Main feature of CPG is the ability to autonomously generate rhythmic activity, even after being disconnected from upper controller or sensory afferents. This distinctive behavior is repeatedly emphasized in literature [10], [13] and shows improbable originating oscillations from positive feedback reaction to external signals. Brain signals only affect the shape of generated patterns and global coordination, as well as provide vestibular signals. Proprioceptive and exteroceptive feedback signals are fundamentally optional, too. They contribute to inhibition of CPG output, resetting its phase, and other conditional events [14].

Apart from investigating the neurophysiology of biological CPG [12], [15], [16], this concept is used in biomechanical engineering for synthesis of control units from neural networks resembling CPG [2], [10], [17], [18] and extensively used in robotics to obtain the same locomotion patterns as in animals [19]-[22].

The application of CPG in muscle-actuated gait control is different from torque-actuated gait in robotics. The latter adds up both flexor and extensor CPG signals to obtain a desired articular value (position, velocity or torque) applied to the PID controller of joint [19], [25], [26]. While the former uses two anti-phase CPG outputs to directly control antagonistic muscles.

Our CPG model was originally inspired by the work of Rybak et al. [23] and combines Rowat and Selverston neuron model [24] with Hebbian plasticity rule [25]. CPG model consists of 2 half-centers with 4 layers: Rhythm Generation (RG), Pattern Formation (PF), Motoneuron (MN), and Sensory Neuron (SN) (Fig. 2). All neurons in this work, except RG, are implemented as sigmoid function. We don't recall the cell equations of neurons here, for mathematical details see [25].

In this work, half-centers of a single CPG create 2 control signals for the opposite groups of muscles: flexion and extension. Half-centers mutually inhibit each other in the first layer. RG layer creates oscillations with frequency adapted from descending signal by the learning rule. Outputs of RG halfcenters are connected to PF neurons, which have 3 functions. First, they integrate exteroceptive signals from ground pressure force (GSN) and body angle (FSN) neurons. Then, PF layer reacts to upper signals from MLR: $\alpha_{M L R}$ and $\theta_{M L R} . \alpha_{M L R}$ changes step length and $\theta_{M L R}$ clamps further propagation of signal, therefore slowing or stopping the gait. And lastly, PF itself non-linearly transforms signal range to $[0 ; 1]$.

MN layer directly controls muscles with inputs from PF layer and proprioceptive muscle sensors from SN layer. In current work, only sensors of type Ia are implemented as they are thought to participate in autonomous gait [14] by limiting joint angles. Each Ia sensory neuron react to positive contraction velocity of its muscle. Which means that Ia sensor indirectly measures opposite muscle's activation velocity. Ia sensor excite its own motoneuron to limit joint's angular velocity.

\section{B. Gait Simulation Platform}

In order to achieve the neuro-musculoskeletal simulation, the model proposed by Rajagopal et al. [1] (Fig. 3, a) has been modified. The original model uses 80 muscles to control leg joints and 17 idealized torque drives for upper body resulting in 37 degrees of freedom (DoF). By using Hill muscle model 

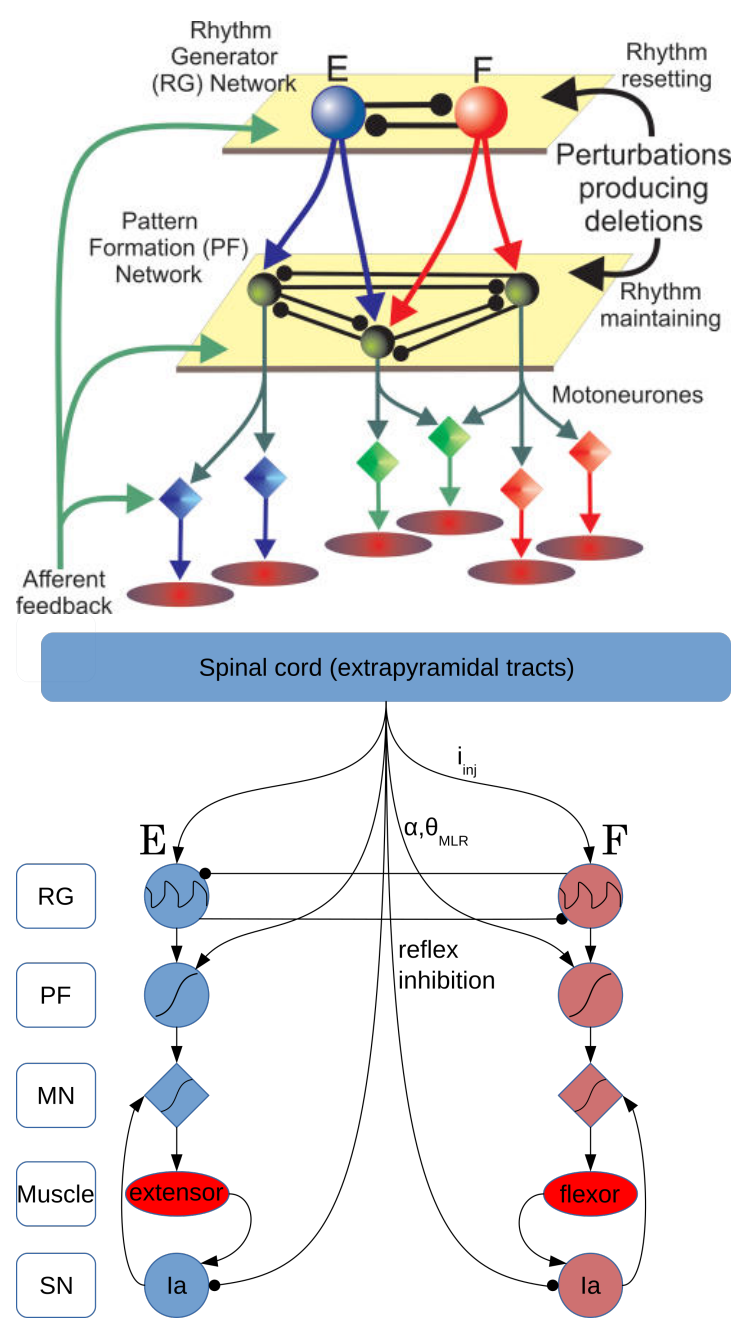

Fig. 2: Top: model of CPG proposed by Rybak et al. [10]. Bottom: proposed CPG controller. Lines ending with arrow and circle stand for excitatory and inhibitory connections respectively. $\mathrm{E}$ and $\mathrm{F}$ stand for extension and flexion halfcenters that control corresponding muscle groups.

with anatomical skeletal geometry, the authors achieved a great reduction of calculation time, while keeping acceptable accuracy of muscle simulation. The model of human body has been developed based on anatomical measurements of 21 corpse and MRI data of 24 healthy people. The authors then verified the model by running forward-time simulation and comparing results to electromyographic and dynamic data of healthy running and walking. The model was a priori scaled to a person using OpenSim built-in tools.

1) Closed-loop Control: Simulation platform is implemented in Python utilizing OpenSim's built-in SWIG wrapper to call musculoskeletal model's C++ functions. Each time-tick the model of CPG network calculates its output from previous state and feedback signals. Each CPG provides excitation values for two corresponding antagonist groups of muscles that are passed to musculoskeletal model. Musculoskeletal model then integrates its state to the next time-tick, producing
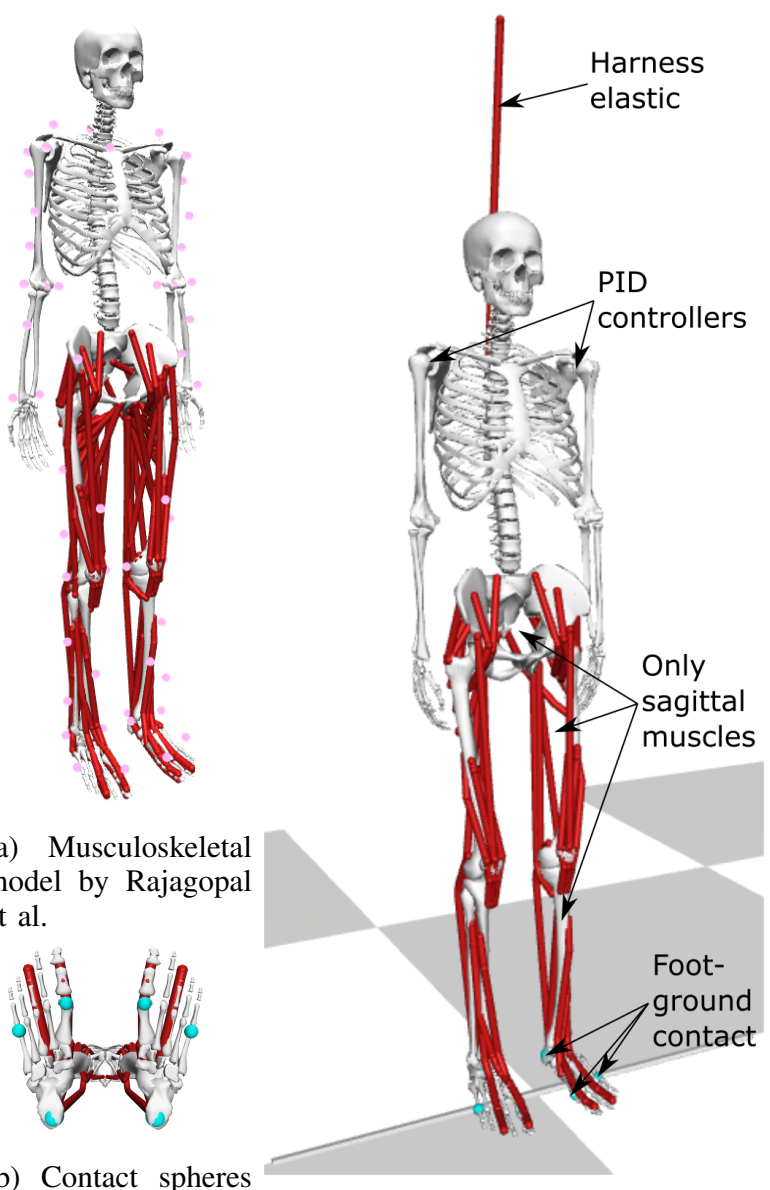

(b) Contact spheres on feet

(c) Modified musculoskeletal model

Fig. 3: (a) The original musculoskeletal model in OpenSim. Pink spheres are marker positions. (b) Position of 6 contact spheres of the feet to interact with the ground. Ground is implemented as half-space. (c) Modified musculoskeletal model has 6 muscle-driven joints of legs and 2 PID-controlled torque-driven shoulder joints.

kinematic and dynamic values of simulated movement [8].

Three of dynamic values are used as feedback signals: muscle stretch velocity for Ia type of proprioceptive signal, ground reaction force for exteroceptive signal, and angle of model in sagittal plane is used as substitution of vestibular signal.

Proprioceptive feedback in this paper consist of Ia-type signals as they affect spinal gait [14]. The role of Ia SN is to prevent excessive joint angular velocity. The sensors measure muscle's positive stretch velocity, i.e. muscle's lengthening. And by this, Ia SN indirectly react to opposite muscle's excessive stretch. Upon activation, Ia SN participates in MN excitation (along with rhythmic PF) to prevent the joint from turning too fast.

Exteroceptive feedback is made up as two types of signals: ground reaction force and global angle of body trunk. Ground reaction force is measured in three contact points on each foot (Fig. 3, b). Reaction force on heel is used for GB neurons and 
force for GF neuron is obtained by summing two forces in toes.

Angle of body trunk is used for FB and FF neurons. FB neuron reacts on positive trunk angle (when model leans backwards) and participates in exciting flexion hip and ankle muscles to prevent falling back. Similarly, FF neuron prevents falling forward by exciting extension muscles.

2) Bio-inspired CPG Model to Control Gait: The CPG network consists of $8 \mathrm{CPGs}$, connected in series (Fig. 1). CPGs in this network are symmetrical between legs, but each CPG in one leg is slightly different in its connections and feedback. The differences are described further.

Hip1 CPG controls two antagonistic groups of muscles: flexor (H1F) and extensor (H1E) that affect only hip joint in sagittal plane. Flexor group consists of iliacus and psoas muscles, while extensor group consists of glutei muscles. This CPG uses both exteroceptor signals: ground and vestibular. And they both are connected to PF layer with their respective weights (Fig. 4, A). Ia receptor of Hip1 from flexion side is also connected to flexion PF neuron of Hip2 to propagate synchronicity from top CPG to bottom ones as described from biological evidence [14].

Hip2 CPG controls muscles that affect both hip and knee joints. Group, denoted as flexor $(\mathrm{H} 2 \mathrm{~F})$, consists only of rectus femoris muscle, which, in fact, acts as flexor for hip and extensor for knee. Similarly, extensor group (H2E) consists of biceps femoris long head, semimembranosus, and semitendinosus muscles, which are extensors for hip and flexors for knee. This CPG uses ground force sensor (Fig. 4, B) and muscle sensor from previous CPG (SH1). They both are connected to PF layer with their respective weights. Ia receptor of Hip2 from flexion side is propagated to both Knee and Ankle CPGs in a more complex manner.

Knee CPG's flexion muscle (KF), gastrocnemius, affect both knee and ankle joints. Knee as flexor and ankle as extensor. But the extensor group of Knee CPG (KE) consists only of knee-affecting muscles: vasti group (intermedius, lateralis, and medialis). This asymmetry increases the complexity of control and is overcome by co-contraction and synergy of muscles. This CPG doesn't use exteroceptor signals but receives twin input from Hip2 CPG's Ia sensor (Fig. 4, C). Flexion half-center $\mathrm{PF}$ receives excitatory input and extension gets inhibitory one [14] from the same $\mathrm{SH} 2$.

Ankle joint dorsiflexion muscles compose flexion $\mathrm{CPG}$ group (AF). They are extensor digitorum longus, extensor hallucis longus, and tibialis anterior muscles. They affect only ankle joint. Plantarflexion muscle in this paper is just soleus, referred to as extension muscle group (AE). Ankle CPG, again, receives both exteroceptor signals in PF layer (Fig. 4, D) and the input from Hip2 CPG's Ia sensor ( $\mathrm{SH} 2$, just for flexion half-center).

3) Modifications to the Musculoskeletal Model in OpenSim: We have modified the original musculoskeletal model to adapt it for our research purpose, forward-time gait simulation (Fig. 3, c).
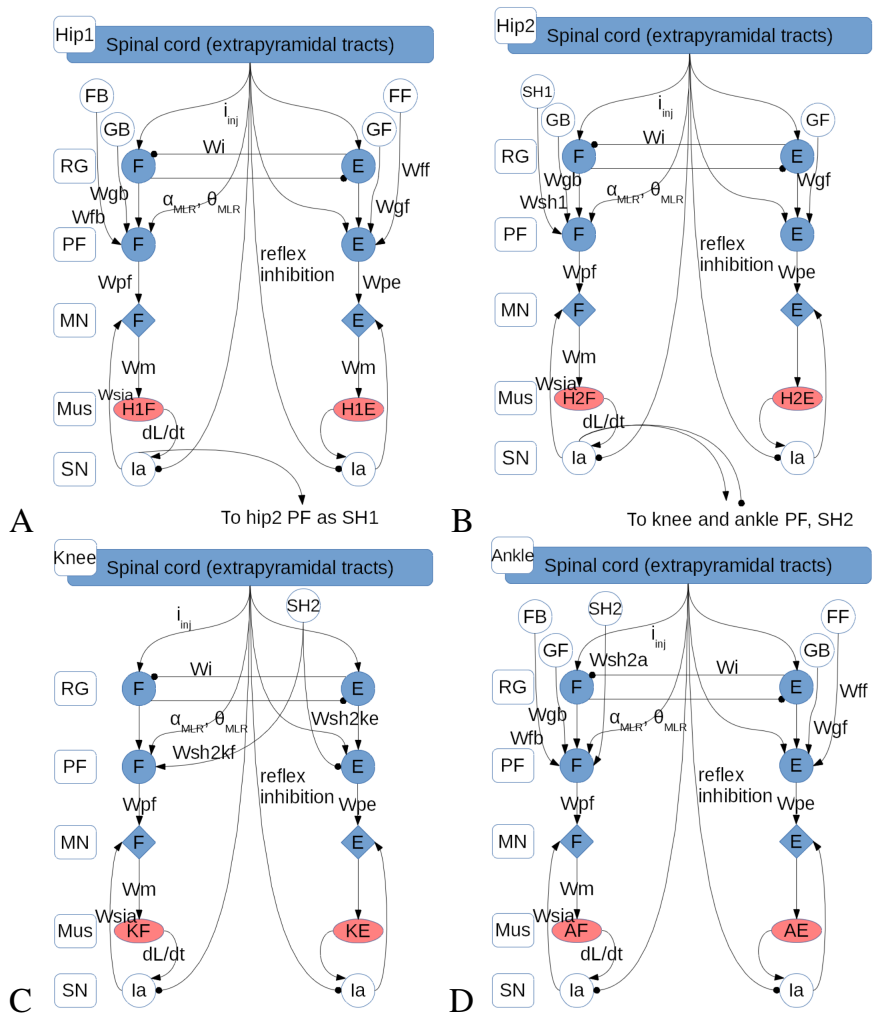

Fig. 4: Details of each CPG. All CPGs contain the same 5 levels: rhythm generation, pattern formation, motoneuron, muscle, and proprioceptive sensors (Ia type). The difference is in used exteroceptors and inter-CPG connections. A: Hip1 CPG scheme for hip-only muscles. B: Hip2 CPG scheme for hip-knee muscles. C: Knee CPG scheme. D: Ankle CPG scheme. Abbreviations: FB, FF are trunk angle neurons; GB, GF ground force neurons; SH1 Ia sensor of Hip1; SH2 Ia sensor of Hip2. Wi are weights of corresponding i neurons. Weight from RG to PF is always 1 . dL/dt is muscle stretch velocity signal. Lines ending with arrow and circle stand for excitatory and inhibitory connections respectively.

Model's modifications can be divided into two groups: (a) simplifying the model; and (b) adding new parts to the model.

a) Model Simplifications: Three modifications were made to simplify the task of gait simulation: restricting model's movements to the sagittal plane; removing muscles that don't contribute to sagittal plane and leaving 44 muscles; and disabling the movements of back and elbow joints.

b) Model's Expansion: We have made some additions to the model to accommodate it to our research. Supportive harness-elastic to prevent body from falling due to the lack of vestibular system simulation in current work. Explicit limitation of leg joint angles to their anatomical capabilities due to lack of soft tissues and skin simulation. And, lastly, six contact spheres on the feet and a ground half-space (Fig. 3b).

4) Optimization of CPG Parameters: In this work, the musculoskeletal model is thought to be unchangeable, but the exact values of CPG parameters and weights of connections 
are in question.

The controller contains 46 variable parameters of four types of CPG. Parameters to control right and left leg are the same. There is a set of 6 parameters, that is present in all CPG types. These are $\alpha$ 's and $\theta$ 's for PF and MN layers and 2 always present weights of connections for Ia sensor (Wsia) and muscle group (Wm). Additionally, there are own sets of parameters in each CPG type, see Fig. 4.

There are not enough insights about functioning of $\mathrm{CPG}$ network, left alone our implementation of neural circuitry. Because of that, the correct way of CPG control is unknown, so the parameters of controller must be evaluated from the overall quality of gait after simulation of several steps.

Current implementation of our forward dynamics simulation is single-threaded, which leaves us with the simulation speed of up to one minute of calculation per second of gait on $4 \mathrm{GHz}$ processor core. Although, any amount of simulations could be arranged in parallel.

This leaves us with the necessity to optimize an expensive target function, when each set of parameters is evaluated for up to ten minutes of calculations. To reduce the parameter search time, we use BlackBox, a Python module for parallel optimization of expensive black-box functions [27].

The target function itself holds the idea to evaluate the overall quality of gait by comparing dynamic of simulation to corresponding human values during gait. In this paper, we use joints angle values for all six joints, extracted from [1].

As for the comparison method, scalar product was chosen for its simplicity and suitability. It requires the equality of length and time-steps of compared signals. The extracted values held only one period of normal gait, therefore required preprocessing before being used. This included equalizing the start and final point, accounting for time resolution, and starting phase.

Finally, the target function returns a single value of $[0 ; 1]$, indicating the similarity of resulting gait to human walk,

\section{RESULTS}

To illustrate the capabilities of gait simulation platform we show two types of walking gait: normal and temporarily interrupted by MLR signal. They both use the same musculoskeletal model and same set of CPG network settings.

\section{A. Normal Gait}

The normal human walking gait was simulated in the course of 6 seconds to show the transition from standing up in double support phase to walk. Fig. 5 shows signals in CPGs of right leg (signals in left CPGs are similar and therefore not given).

All RG neurons have the same frequency, but different initial phase to account for normal muscle activation timing. RG output, together with all exteroceptive signals (SNF, SNG, $\mathrm{SH} 1$ or SH2), comes to PF layer. The latter's output is further combined with SN Ia inside MN layer to excite muscles.

Muscles' resulting activation values are shown on Fig. 7. The parameters of CPG controller were found as local optimum during an optimization procedure and therefore are the subject of discussion.

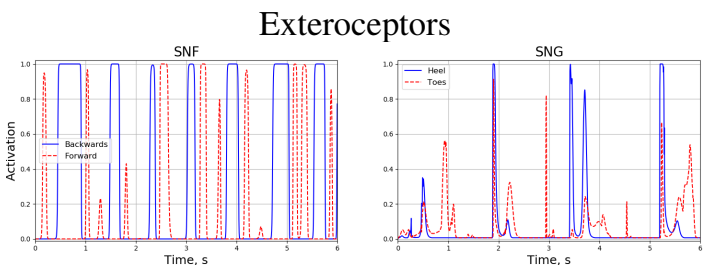

Hip1
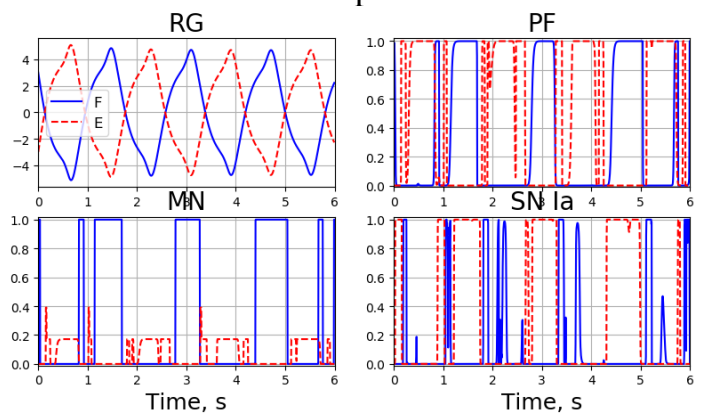

Hip2
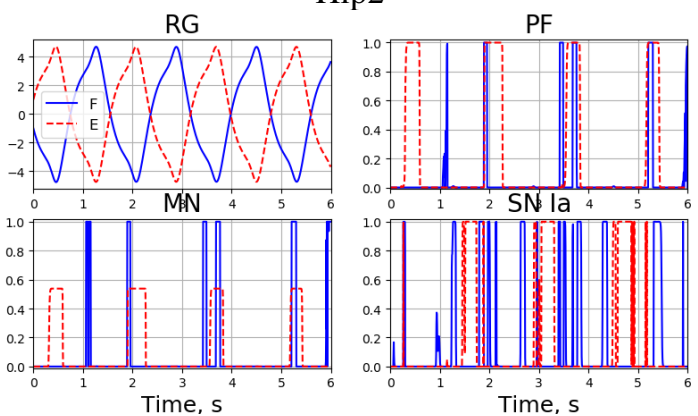

Knee
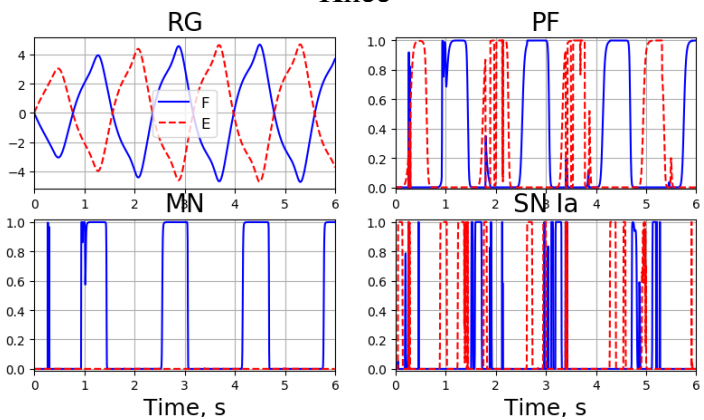

Ankle
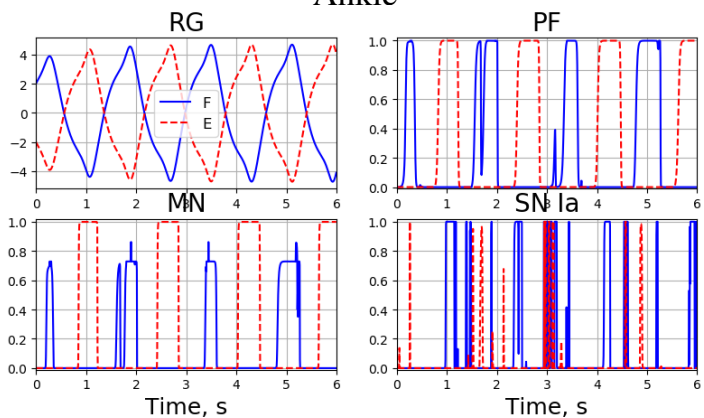

Fig. 5: Signals in right CPGs during normal gait. F (solid blue) and $\mathrm{E}$ (dashed red) stand for flexion and extension. First row: SNF is trunk angle sensor; SNG is ground force sensor; SN Ia is muscle stretch velocity sensor. Second row: outputs of three CPG layers. MN output works as muscle excitation. 


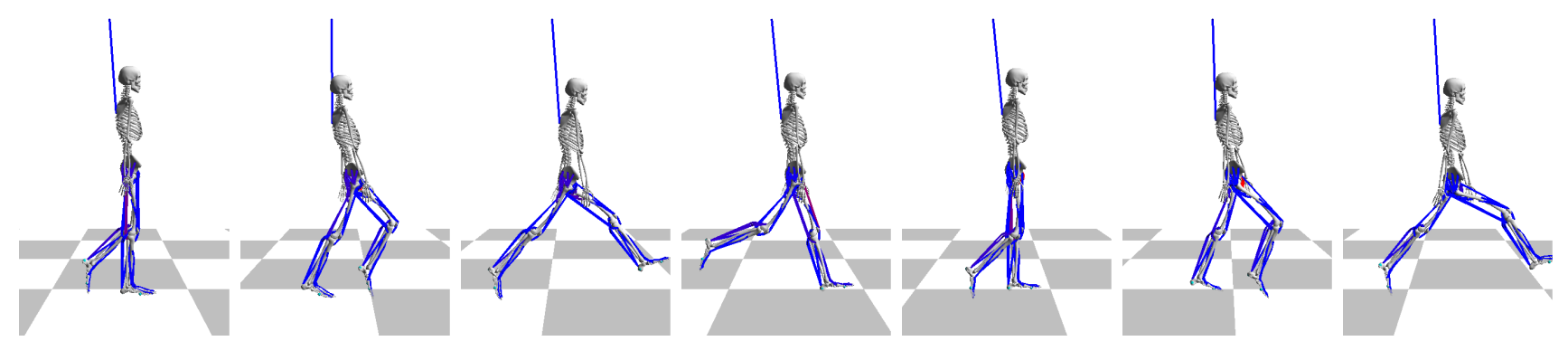

Fig. 6: Several frames of simulated walking gait of second cycle in OpenSim. Time between frames is 0.2 seconds. Color of muscles denote their activation, from blue as 0 to red as 1. On top, connected to model's back, is supportive harness-elastic.
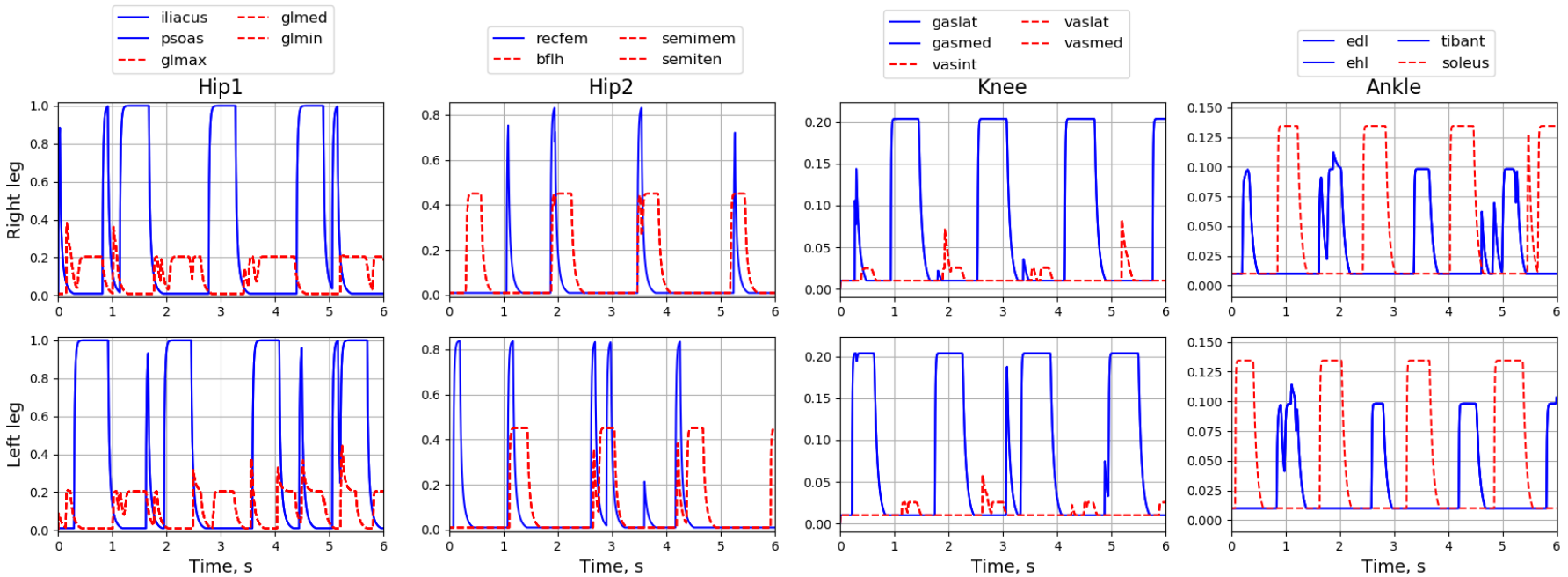

Fig. 7: Muscles activation and corresponding muscles group (on top).
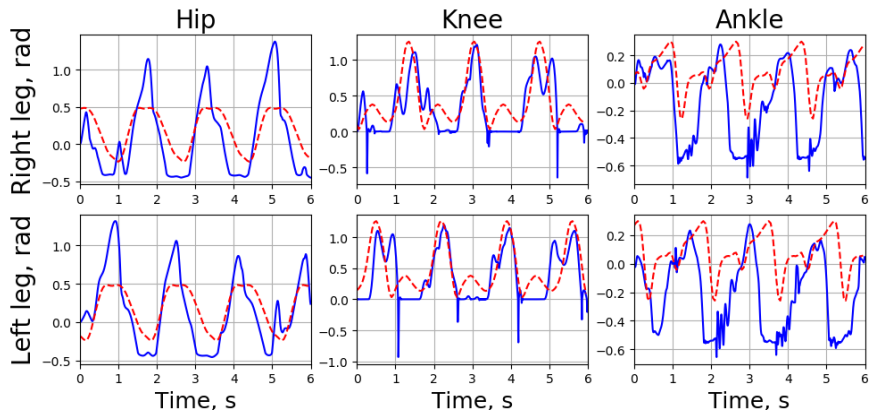

Fig. 8: Joint angles during normal gait. Dashed lines are the normal human joint angles, extracted and modified from [1].

Muscle actuation enables the model to walk. The joint angles are shown on Fig. 8. Very brief negative knee angle values do not affect the gait and are the result of energy dissipation in OpenSim for the case of joint angle limits, and are not observed on simulation animation. Several frames of animation are shown on Fig. 6.

The shape of simulated joint angles in hip and knee is largely similar to human angles, although ankle is different. Higher amplitude of hip angle is required because of ankles, to remove model's stumbling to ground. Difference between

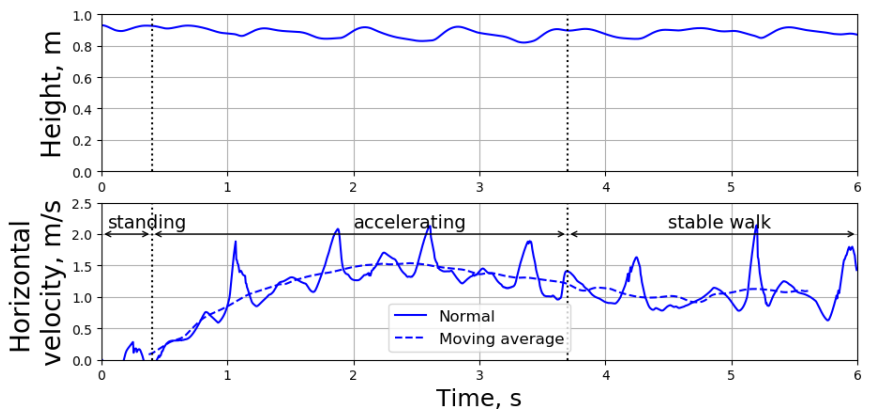

Fig. 9: Top: pelvis height. Bottom: pelvis velocity. Dashed line shows moving average of velocity with window of 0.75 seconds.

human ankle behavior and simulated one may come from the difficulty to produce a realistic model of the foot (number of muscles, sole flexibility) and thus to simulate correctly the foot/ground contact. In the OpenSim model, the foot's sole is almost rigid and creates some changes in the joint angle behavior compared to the human [29].

Fig. 9 shows two main characteristics of the gait, pelvis height and horizontal velocity. Pelvis height oscillates for 10 $\mathrm{cm}$ due to stepping, while human values are about $8 \mathrm{~cm} \mathrm{[28].}$ 

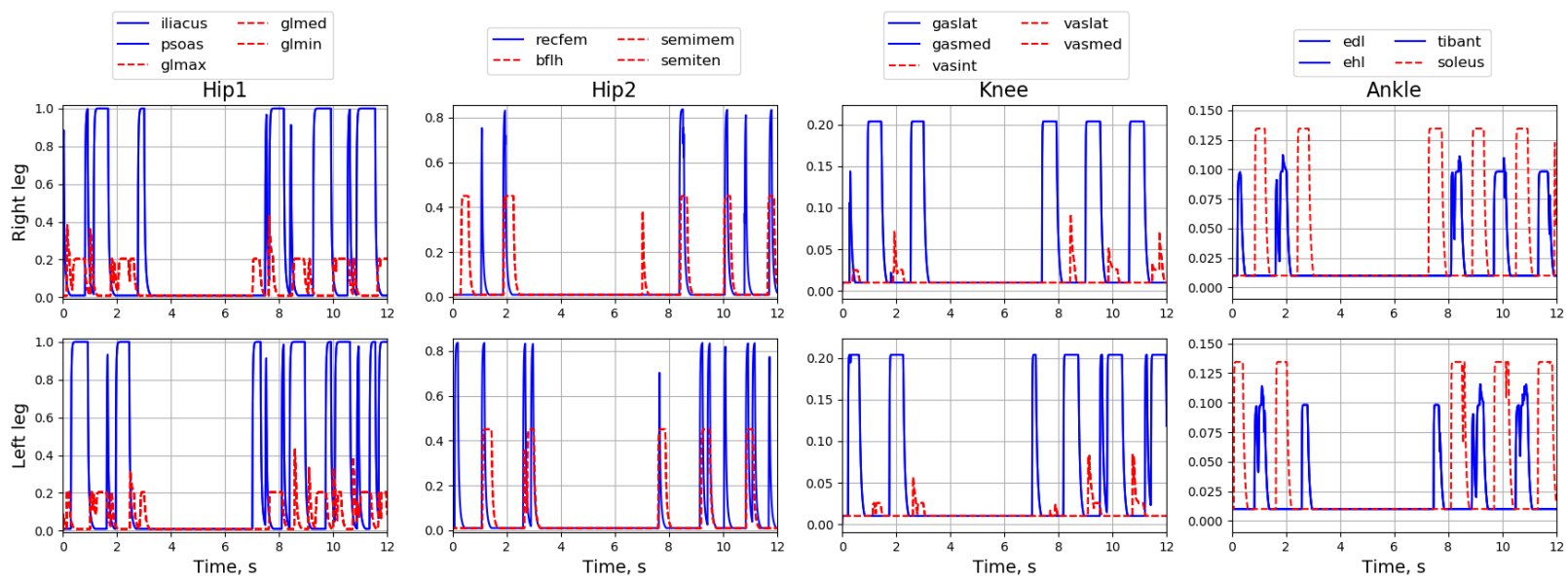

Fig. 10: Muscle activation during disrupted gait. $\theta_{M L R}$ signal clamp CPG control from $3^{\text {rd }}$ to $7^{\text {th }}$ second.
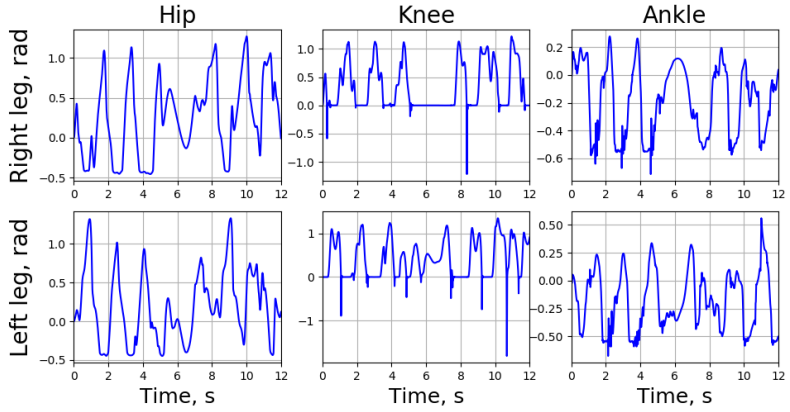

Fig. 11: Joint angles during disrupted gait. $\theta_{M L R}$ signal clamp CPG control from $3^{\text {rd }}$ to $7^{\text {th }}$ second.

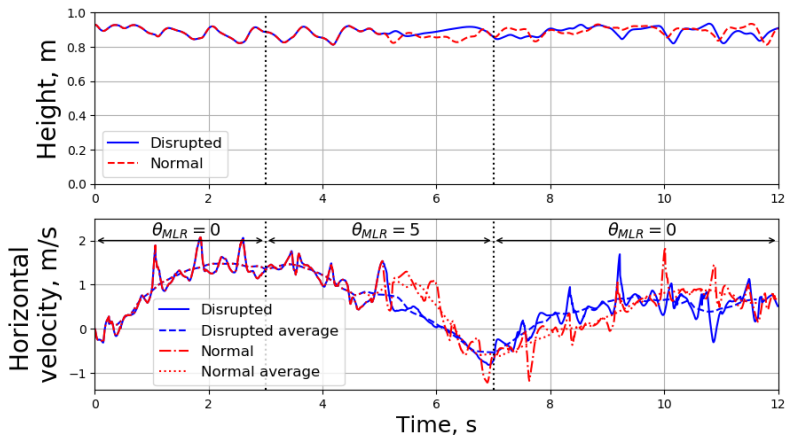

Fig. 12: Normal and disrupted pelvis height and velocity with moving averages.

Horizontal velocity of pelvis and its moving average show the transition from standing position to walk during first 2 seconds.

\section{B. Disrupted Gait}

Abnormal gait is simulated with rising the $\theta_{M L R}$ signal that clamps CPG control from PF layer and further. This gait was simulated for 12 seconds to show model's motor function stop and release. $\theta_{M L R}=5$ between $3^{r d}$ and $7^{\text {th }}$ second. The rest of time $\theta_{M L R}=0$.
The absence of signal from motoneurons results in no muscle activation (Fig. 10). In this state, only the supportive harness-elastic prevents model from falling due to lack of reflexes and vestibular system. Its top end moves above model's center of mass. During the disrupted state, joint angles follow the overall body position provided by support. As relaxed body tries to fall backwards, hip angle stays on 0.5 radiant, knee extends to straight posture, and ankle is plantarflexed (Fig. 11).

After the release of CPG signal on $7^{\text {th }}$ second, controller output resumes to oscillate and restarts gait, that is shown on Fig. 12. It's important to note, that normal gait stumbles on $6^{\text {th }}$ second with this parameter set. Disrupted model moves inertially for a second before stop and for another two seconds, model gains velocity until the average of $1 \mathrm{~m} / \mathrm{s}$, defined by CPG frequency and weights.

This effect of $\theta_{M L R}$ can be considered like a freezing of gait, observed in parkinsonian patients. Indeed, Parkinson's is a neurodegenerative disease that originates in basal ganglia region that innervates MLR. Thus, the simulation presented here can be seen as the first step toward a more complex modelling platform of cerebral-spinal links.

Nevertheless, it can be observed that during freezing of gait the modelled human body cannot support itself for standing up and the elastic acts like a real medical harness. This problem could be avoided by adding to each CPG specific neurons able to produce tonic activity for muscles in order to control the standing posture and balance.

\section{CONCLUSION}

This paper presents a new neuro-musculoskeletal gait simulation platform based on muscle-actuated lower human body in OpenSim and an original model of central pattern generator able to generate variable rhythmic signals. Forward dynamics simulations show the ability of our model to simulate a normal healthy gait and a walk interrupted by a sudden stop. Both gaits are controlled by bio-inspired closed-loop CPG-based circuitry.

Musculoskeletal model contains three joints per leg controlled by 44 Hill-type muscle models activated by network 
of 8 models of central pattern generators. Output signals of motoneurons are applied to excitation inputs of modelled muscles of the human lower limbs model.

Each CPG consists of four layers and two types of neurons. Rhythmic neurons are based on the work of Rybak et al. and combine Rowat and Selverston neuron model with Hebbian plasticity rule. Created walking gaits can be changed by variation of intrinsic neural parameters. These variations can be controlled by signals coming from an upper level circuitry.

Our platform for gait simulation is a notable step forward in reaching the goal of simulating abnormal gaits from MLR. These gaits include neurodegenerative diseases, such as Parkinson's.

The ability to simulate various gaits opens a possibility of medical application of our simulation platform, both in research and in diagnosis and treatment planning. Research may benefit from gait analysis and simulation of unobtainable or invasive data and treatment specialists could use our platform to predict interventions in musculoskeletal and mesoscopic nervous system.

Further work includes more computational experiments with different gaits. We have plans to develop a stable human standing by implementing reflexes. This will allow us to get rid of supportive elastic and simulate missing gait features. Additionally, there are possibilities to make this simulator three-dimensional and return removed muscles. Another future goal is to connect an advanced gait decision-making model of MLR to our platform.

\section{ACKNOWLEDGMENT}

This work is supported by Erasmus program between National Technical University of Ukraine "Igor Sikorsky Kyiv Polytechnic Institute" and Université de Lorraine, and French embassy of Ukraine.

\section{REFERENCES}

[1] A. Rajagopal, C. L. Dembia, M. S. DeMers, D. D. Delp, J. L. Hicks, and S. L. Delp, "Full-Body Musculoskeletal Model for Muscle-Driven Simulation of Human Gait," IEEE Trans Biomed Eng, vol. 63, no. 10, pp. 2068-2079, 2016.

[2] K. Hase, K. Miyashita, S. Ok, and Y. Arakawa, "Human gait simulation with a neuromusculoskeletal model and evolutionary computation," J. Visual. Comput. Animat., vol. 14, no. 2, pp. 73-92, May 2003.

[3] K. Matsuoka, "Sustained oscillations generated by mutually inhibiting neurons with adaptation," Biological Cybernetics, vol. 52, no. 6, pp. 367-376, Oct. 1985.

[4] Y Kim, Y Tagawa, G. Obinata, K. Hase., Robust control of CPG-based 3D neuromusculoskeletal walking model, Biol Cybern (2011) 105: 269. https://doi.org/10.1007/s00422-011-0464-4

[5] D. Zhang, P. Poignet, A. P. L. Bo and W. T. Ang, "Exploring Peripheral Mechanism of Tremor on Neuromusculoskeletal Model: A General Simulation Study," in IEEE Transactions on Biomedical Engineering, vol. 56, no. 10, pp. 2359-2369, Oct. 2009. doi: 10.1109/TBME.2009.2023979

[6] K. Jansen, F. De Groote, W. Aerts, J. De Schutter, J. Duysens and I. Jonkers, "Altering length and velocity feedback during a neuromusculoskeletal simulation of normal gait contributes to hemiparetic gait characteristics," in Journal of NeuroEngineering and Rehabilitation 2014 11:78. doi:10.1186/1743-0003-11-78
[7] A. Murai, K. Yamane and Y. Nakamura, "Modeling and identification of human neuromusculoskeletal network based on biomechanical property of muscle," 30th Annual International Conference of the IEEE Engineering in Medicine and Biology Society, Vancouver, BC, 2008, pp. 3706-3709. doi: 10.1109/IEMBS.2008.4650014

[8] S. L. Delp et al., "OpenSim: open-source software to create and analyze dynamic simulations of movement," IEEE Trans Biomed Eng, vol. 54, no. 11, pp. 1940-1950, Nov. 2007.

[9] A. Shachykov, P. Hénaff, A. Popov, and A. Shulyak, "CPG-based circuitry for controlling musculoskeletal model of human locomotor system," in 2017 IEEE Biomedical Circuits and Systems Conference (BioCAS), 2017, pp. 1-4.

[10] I. A. Rybak, N. A. Shevtsova, M. Lafreniere-Roula, and D. A. McCrea, "Modelling spinal circuitry involved in locomotor pattern generation: insights from deletions during fictive locomotion,” J Physiol, vol. 577, no. Pt 2, pp. 617-639, Dec. 2006.

[11] M. MacKay-Lyons, "Central pattern generation of locomotion: a review of the evidence," Phys Ther, vol. 82, no. 1, pp. 69-83, Jan. 2002.

[12] O. Kiehn and K. Dougherty, "Locomotion: Circuits and Physiology," in Neuroscience in the 21st Century, D. W. Pfaff, Ed. New York, NY: Springer New York, 2013, pp. 1209-1236.

[13] T. G. Brown, "On the nature of the fundamental activity of the nervous centres; together with an analysis of the conditioning of rhythmic activity in progression, and a theory of the evolution of function in the nervous system,” J Physiol, vol. 48, no. 1, pp. 18-46, Mar. 1914.

[14] S. Rossignol, R. Dubuc, and J.-P. Gossard, "Dynamic sensorimotor interactions in locomotion," Physiol. Rev., vol. 86, no. 1, pp. 89-154, Jan. 2006.

[15] E. Marder and D. Bucher, "Central pattern generators and the control of rhythmic movements," Current Biology, vol. 11, no. 23, pp. R986R996, Nov. 2001.

[16] P. A. Guertin, "The mammalian central pattern generator for locomotion,” Brain Research Reviews, vol. 62, no. 1, pp. 4556, Dec. 2009.

[17] P. F. Rowat and A. I. Selverston, "Learning algorithms for oscillatory networks with gap junctions and membrane currents," Network: Computation in Neural Systems, vol. 2, no. 1, pp. 17-41, Jan. 1991.

[18] A. J. Ijspeert, "Central pattern generators for locomotion control in animals and robots: A review," Neural Networks, vol. 21, no. 4, pp. 642-653, May 2008.

[19] Nachstedt, T., Tetzlaff, C., and Manoonpong, P. (2017). Fast dynamical coupling enhances frequency adaptation of oscillators for robotic locomotion control. Frontiers in neurorobotics 11

[20] Yu, J., Tan, M., Chen, J., and Zhang, J. (2014). A survey on cpg-inspired control models and system implementation. IEEE Transactions on neural networks and learning systems, 25, 441-456

[21] Taga, G. (1998). A model of the neuro-musculo-skeletal system for anticipatory adjustment of human locomotion during obstacle avoidance. Biological cybernetics 78, 9-17

[22] Arikan, K. B. and Irfanoglu, B. (2011). A test bench to study bioinspired control for robot walking. Journal of Control Engineering and Applied Informatics $13,76-80$

[23] I. A. Rybak, K. J. Dougherty, and N. A. Shevtsova, "Organization of the Mammalian Locomotor CPG: Review of Computational Model and Circuit Architectures Based on Genetically Identified Spinal Interneurons," eNeuro, vol. 2, no. 5, p., Sep. 2015.

[24] P. F. Rowat and A. I. Selverston, "Oscillatory Mechanisms in Pairs of Neurons Connected with Fast Inhibitory Synapses," J Comput Neurosci, vol. 4, no. 2, pp. 103-127, Apr. 1997.

[25] M. Jouaiti, L. Caron, and P. Hénaff, "Hebbian plasticity in CPG controllers facilitates self-synchronization for human-robot handshaking", Frontiers in Neurorobotics, Vol. 12 , pp. 29, DOI=10.3389/fnbot.2018.00029.

[26] T. Geng, B. Porr, and F. Wörgötter, "Fast Biped Walking with a Sensordriven Neuronal Controller and Real-time Online Learning," The Int'l Journal of Robotics Research, vol. 25, no. 3, pp. 243-259, Mar. 2006.

[27] P. Knysh and Y. Korkolis, "Blackbox: A procedure for parallel optimization of expensive black-box functions," arXiv:1605.00998 [cs, math], May 2016.

[28] L. Zhao, L. Zhang, L. Wang, and J. Wang, "Three-dimensional motion of the pelvis during human walking," in IEEE International Conference Mechatronics and Automation, 2005, 2005, vol. 1, pp. 335-339 Vol. 1.

[29] H. Serhan and P. Hénaff, "MuscleLike Compliance in Knee Articulations Improves Biped Robot Walkings,' Recent Advances in Robotic Systems, 2016. 